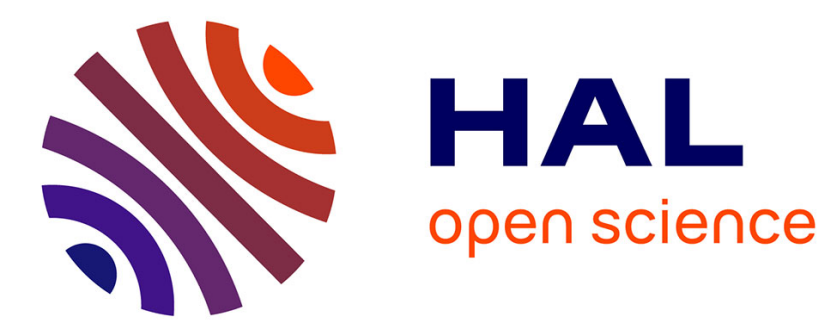

\title{
On analysis of Persidskii systems and their implementations using LMIs
}

Denis Efimov, Alexander Aleksandrov

\section{To cite this version:}

Denis Efimov, Alexander Aleksandrov. On analysis of Persidskii systems and their implementations using LMIs. Automatica, In press. hal-03291855

\section{HAL Id: hal-03291855 \\ https://inria.hal.science/hal-03291855}

Submitted on 19 Jul 2021

HAL is a multi-disciplinary open access archive for the deposit and dissemination of scientific research documents, whether they are published or not. The documents may come from teaching and research institutions in France or abroad, or from public or private research centers.
L'archive ouverte pluridisciplinaire HAL, est destinée au dépôt et à la diffusion de documents scientifiques de niveau recherche, publiés ou non, émanant des établissements d'enseignement et de recherche français ou étrangers, des laboratoires publics ou privés. 


\title{
On analysis of Persidskii systems and their implementations using LMIs
}

\author{
Denis Efimov ${ }^{\mathrm{a}, \mathrm{c}}$ Alexander Aleksandrov ${ }^{\mathrm{b}}$ \\ ${ }^{\mathrm{a}}$ Inria, Univ. Lille, CNRS, UMR 9189 - CRIStAL, F-59000 Lille, France \\ ${ }^{\mathrm{b}}$ Saint Petersburg State University, 7-9 Universitetskaya nab., 199034 Saint Petersburg, Russia \\ ${ }^{\mathrm{c}}$ ITMO University, 197101 Saint Petersburg, Russia
}

\begin{abstract}
The conditions of (integral) input-to-state stability and input-output-to-state stability are established for a class of generalized Persidskii systems. The proposed conditions are formulated using linear matrix inequalities. Based on these results the conditions of convergence are derived for discretizations of this class of models obtained by the explicit and the implicit Euler methods. The proposed theory is finally applied to design a robust stabilization control.
\end{abstract}

\section{Introduction}

Control design and stability analysis for dynamical systems are complex problems, especially in the nonlinear case. Answering these issues, many concepts and methods have been proposed in the theory of systems and controls (Khalil, 1996). In the nonlinear framework, the only available approach for stability check is the Lyapunov function method (Lyapunov, 1992; Malkin, 1952; Hahn, 1967) and the related extensions (LaSalle and Lefchetz, 1961; van der Schaft, 1996; Lin et al., 1996; Bacciotti and Rosier, 2005). The shortage of this approach is the lack of constructive techniques that assign Lyapunov functions with desired properties to generic nonlinear systems. The existing solutions for nonlinear dynamics usually deal with various canonical forms: Lurie systems, Lipschitz dynamics, Persidskii models, homogeneous systems, etc. The design of Lyapunov functions for the first three mentioned classes of systems is based on closeness of these models to the linear ones, where the analysis and design theories are rather complete.

In the present paper the focus is put on a stratum of Persidskii systems. This class of models was first introduced for stability analysis in (Barbashin, 1961), where a linear combination of the integrals of the nonlinearities was used as a Lyapunov function. Next, that result was extended by

\footnotetext{
* This work is partially supported by the Ministry of Science and Higher Education of Russian Federation, passport of goszadanie no. 2019-0898 and by the Russian Foundation for Basic Research (grant no. 19-01-00146-a).
}

Persidskii in (Persidskii, 1969), where he augmented the Lyapunov function by a combination of the absolute values of the states. Further, this class of nonlinear models was studied in the context of diagonal stability (Kazkurewicz and Bhaya, 1999; Ferreira et al., 2005), opinion dynamics (Altafini, 2013), sliding mode control (Hsu et al., 2000; Aparicio et al., 2019; Zhang, 2020), Lur'e systems (Arcak and Teel, 2002), neural networks (Hopfield and Tank, 1986; Sontag, 1993; Karny et al., 1998) and in other applications (Erickson and Michel, 1985). In this work these results will be developed to robust stability analysis of a more general form of the dynamics enlarging the domain of possible applications.

A popular and extremely useful framework for robust stability analysis of nonlinear dynamical systems is presented by the input-to-state stability theory (Sontag, 2001; Dashkovskiy et al., 2011). This methodology suggests a variety of tools allowing the robust stability of nonlinear systems to be evaluated with respect to external inputs and taking into account the outputs. It also allows the influence of different sources of uncertainty to be assessed in the applications.

In this work, we introduce a class of generalized Persidskii systems, which have a linear part and different nonlinearities $^{1}$. Next, a special structure of Lyapunov function is proposed, and it is demonstrated that its properties and

\footnotetext{
1 A preliminary version of this work (Efimov and Aleksandrov, 2019) does not contain proofs, while focusing on another application.
} 
the characteristics of its derivative can be constrained by solving a series of linear matrix inequalities (LMIs), to this end a fine manipulation of cross-terms in the Lyapunov function and its derivative is effectuated utilizing the features of nonlinearities. It is also shown that the discretization of this class of nonlinear dynamical systems by using the explicit or implicit Euler methods preserves the convergence of trajectories under mild restrictions (for nonlinear systems it is not always the case (Efimov et al., 2017, 2019)). Finally, an application of the proposed theory to a robust control design is given to illustrate its efficacy: we consider a linear nominal model subject to a nonlinearity hidden in the disturbance, and the imposed upper bound on the disturbance does not allow the problem to be solved by a linear feedback.

The outline of this paper is as follows. Preliminary results and notions are introduced in Section 2. The problem statement is given in Section 3. Robust stability conditions are established in Section 4. The Euler discretization properties are studied in Section 5. A control design method is presented in Section 6.

\section{Notation}

- $\mathbb{R}_{+}=\{x \in \mathbb{R}: x \geq 0\}$, where $\mathbb{R}$ is the set of real number.

- $|\cdot|$ denotes the absolute value in $\mathbb{R},\|\cdot\|$ denotes the Euclidean norm on $\mathbb{R}^{n}$.

- For a (Lebesgue) measurable function $d: \mathbb{R}_{+} \rightarrow \mathbb{R}^{m}$ define the norm $\|d\|_{\left[t_{0}, t_{1}\right)}=\operatorname{ess}_{\sup _{t \in\left[t_{0}, t_{1}\right)}}\|d(t)\|$, then $\|d\|_{\infty}=\|d\|_{[0,+\infty)}$ and the set of $d(t)$ with the property $\|d\|_{\infty}<+\infty$ we further denote as $\mathcal{L}_{\infty}^{m}$ (the set of essentially bounded measurable functions).

- A continuous function $\alpha: \mathbb{R}_{+} \rightarrow \mathbb{R}_{+}$belongs to the class $\mathcal{K}$ if $\alpha(0)=0$ and the function is strictly increasing. The function $\alpha: \mathbb{R}_{+} \rightarrow \mathbb{R}_{+}$belongs to the class $\mathcal{K}_{\infty}$ if $\alpha \in \mathcal{K}$ and it is increasing to infinity. A continuous function $\beta: \mathbb{R}_{+} \times \mathbb{R}_{+} \rightarrow \mathbb{R}_{+}$belongs to the class $\mathcal{K} \mathcal{L}$ if $\beta(\cdot, t) \in \mathcal{K}$ for each fixed $t \in \mathbb{R}_{+}$and $\beta(s, \cdot)$ is decreasing to zero for each fixed $s \in \mathbb{R}_{+}$.

- The notation $D V(x) f(x)$ stands for the directional derivative of a continuously differentiable function $V$ with respect to the vector field $f$ evaluated at the point $x$.

- Denote the identity matrix of dimension $n \times n$ by $I_{n}$, the vector of dimension $n$ with all elements equal 1 by $1_{n}$.

- A finite series of integers $1,2, \ldots, n$ is denoted by $\overline{1, n}$.

- $\operatorname{diag}\{g\} \in \mathbb{D}^{n}$ represents a diagonal matrix of dimension $n \times n$ with a vector $g \in \mathbb{R}^{n}$ on the main diagonal, where $\mathbb{D}^{n} \subset \mathbb{R}^{n \times n}$ is the set of diagonal matrices. The set of diagonal matrices of dimension $n \times n$ with nonnegative elements will be denoted by $\mathbb{D}_{+}^{n}=\mathbb{D}^{n} \cap \mathbb{R}_{+}^{n \times n}$. For $\Lambda \in$ $\mathbb{D}_{+}^{n}, \Lambda_{i}$ with $i=\overline{1, n}$ corresponds to the $i^{\text {th }}$ element on the main diagonal.

- For a symmetric matrix $P \in \mathbb{R}^{n \times n}$, the relations $P>0$ or $P \geq 0$ mean that it is positive definite or semi-definite, respectively.

\section{Preliminaries}

Consider a nonlinear system:

$$
\dot{x}(t)=f(x(t), d(t)), t \geq 0,
$$

where $x(t) \in \mathbb{R}^{n}$ is the state, $d(t) \in \mathbb{R}^{m}$ is the external input, $d \in \mathcal{L}_{\infty}^{m}$, and $f: \mathbb{R}^{n+m} \rightarrow \mathbb{R}^{n}$ is a locally Lipschitz (or Hölder) continuous function, $f(0,0)=0$. In some cases the system (1) is equipped with an output $y(t) \in \mathbb{R}^{p}$ :

$$
y(t)=h(x(t)),
$$

where $h: \mathbb{R}^{n} \rightarrow \mathbb{R}^{p}$ is a continuous function. For an initial condition $x_{0} \in \mathbb{R}^{n}$ and input $d \in \mathcal{L}_{\infty}^{m}$, define the corresponding solutions by $x\left(t, x_{0}, d\right)$ for any $t \geq 0$ for which the solution exists, $y\left(t, x_{0}, d\right)=h\left(x\left(t, x_{0}, d\right)\right)$.

In this work we will be interested in the following stability properties (Sontag, 2001; Dashkovskiy et al., 2011):

Definition 1 The system (1) is called input-to-state practically stable (ISpS), if there are functions $\beta \in \mathcal{K} \mathcal{L}, \gamma \in \mathcal{K}$ and a constant $c \geq 0$ such that

$$
\left\|x\left(t, x_{0}, d\right)\right\| \leq \beta\left(\left\|x_{0}\right\|, t\right)+\gamma\left(\|d\|_{[0, t)}\right)+c \quad \forall t \geq 0
$$

for any input $d \in \mathcal{L}_{\infty}^{m}$ and any $x_{0} \in \mathbb{R}^{n}$. The function $\gamma$ is called nonlinear asymptotic gain. The system is called input-to-state stable (ISS) if $c=0$.

Definition 2 The system (1) is called integral ISS (iISS), if there are functions $\alpha \in \mathcal{K}_{\infty}, \gamma \in \mathcal{K}$ and $\beta \in \mathcal{K} \mathcal{L}$ such that for any $x_{0} \in \mathbb{R}^{n}$ and $d \in \mathcal{L}_{\infty}^{m}$ the estimate holds:

$$
\alpha\left(\left\|x\left(t, x_{0}, d\right)\right\|\right) \leq \beta\left(\left\|x_{0}\right\|, t\right)+\int_{0}^{t} \gamma(\|d(s)\|) d s \quad \forall t \geq 0 .
$$

The previous property introduced in (Sontag, 1998) is close to the integral stability notion given in (Halanay, 1966).

Definition 3 The system (1), (2) with $d=0$ is called output-to-state stable (OSS), if there are functions $\beta \in \mathcal{K} \mathcal{L}$ and $\gamma \in \mathcal{K}$ such that for all $x_{0} \in \mathbb{R}^{n}$

$\left\|x\left(t, x_{0}, 0\right)\right\| \leq \beta\left(\left\|x_{0}\right\|, t\right)+\gamma\left(\|y\|_{[0, t)}\right) \quad \forall t \in\left[0, t_{\max }\left(x_{0}\right)\right)$.

In this definition $t_{\max }\left(x_{0}\right) \leq+\infty$ determines the interval of existence of $x\left(t, x_{0}, 0\right)$ for the system (1), (2).

Definition 4 The system (1), (2) is called input-outputto-state stable (IOSS) if there are functions $\beta \in \mathcal{K} \mathcal{L}$ and $\gamma_{1}, \gamma_{2} \in \mathcal{K}$ such that for all $x_{0} \in \mathbb{R}^{n}, d \in \mathcal{L}_{\infty}^{m}$ and all $t \in\left[0, t_{\max }\left(x_{0}, d\right)\right)$,

$$
\left\|x\left(t, x_{0}, d\right)\right\| \leq \beta\left(\left\|x_{0}\right\|, t\right)+\gamma_{1}\left(\|d\|_{[0, t)}\right)+\gamma_{2}\left(\|y\|_{[0, t)}\right) .
$$

Here again $t_{\max }\left(x_{0}, d\right) \leq+\infty$ defines the interval of existence of solutions for the system (1), (2).

These properties have the following characterizations in terms of existence of Lyapunov functions: 
Definition $5 A$ smooth function $V: \mathbb{R}^{n} \rightarrow \mathbb{R}_{+}$is called ISpS-Lyapunov function for the system (1) if there are $r \geq$ $0, \alpha_{1}, \alpha_{2}, \alpha_{3} \in \mathcal{K}_{\infty}$ and $\theta \in \mathcal{K}$ such that

$$
\begin{gathered}
\alpha_{1}(\|x\|) \leq V(x) \leq \alpha_{2}(\|x\|), \\
D V(x) f(x, d) \leq r+\theta(\|d\|)-\alpha_{3}(\|x\|)
\end{gathered}
$$

for all $x \in \mathbb{R}^{n}$ and all $d \in \mathbb{R}^{m}$. Such a function $V$ is called ISS-Lyapunov function if $r=0$, and it is iISS-Lyapunov function if $\alpha_{3}: \mathbb{R}_{+} \rightarrow \mathbb{R}_{+}$is just a positive definite function.

Note that an ISS-Lyapunov function can also satisfy the following equivalent condition for some $\chi \in \mathcal{K}$ :

$$
\|x\|>\chi(\|d\|) \Rightarrow D V(x) f(x, d) \leq-\alpha_{3}(\|x\|) .
$$

Definition 6 A smooth function $V: \mathbb{R}^{n} \rightarrow \mathbb{R}_{+}$is called OSS-Lyapunov function for the system (1), (2) with $d=0$ if there are functions $\alpha_{1}, \alpha_{2}, \alpha_{3} \in \mathcal{K}_{\infty}$ and $\sigma \in \mathcal{K}$ such that for all $x \in \mathbb{R}^{n}$ :

$$
\begin{gathered}
\alpha_{1}(\|x\|) \leq V(x) \leq \alpha_{2}(\|x\|), \\
D V(x) f(x, 0) \leq-\alpha_{3}(\|x\|)+\sigma(\|y\|) .
\end{gathered}
$$

Definition $7 A$ smooth function $V: \mathbb{R}^{n} \rightarrow \mathbb{R}_{+}$is called IOSS-Lyapunov function for the system (1), (2) if for some functions $\alpha_{1}, \alpha_{2}, \alpha_{3} \in \mathcal{K}_{\infty}$ and $\sigma_{1}, \sigma_{2} \in \mathcal{K}$ the inequalities

$$
\begin{gathered}
\alpha_{1}(\|x\|) \leq V(x) \leq \alpha_{2}(\|x\|), \\
D V(x) f(x, d) \leq-\alpha_{3}(\|x\|)+\sigma_{1}(\|d\|)+\sigma_{2}(\|y\|)
\end{gathered}
$$

hold for all $x \in \mathbb{R}^{n}$ and all $d \in \mathbb{R}^{m}$.

The relations between these Lyapunov characterizations and the robust stability properties are given below:

Theorem 1 The system (1) is ISS (ISpS, iISS) iff it admits an ISS (ISpS, iISS)-Lyapunov function.

Theorem 2 The system (1), (2) is IOSS (OSS for $d=0$ ) iff it admits an IOSS (OSS)-Lyapunov function.

The sufficient part of these theorems is valid under simple continuity of (1). A consequence of Theorem 1 and Definition 5 is that an ISS system (1) is also iISS. Another consequence of these theorems is that any ISS/IOSS/OSS system belongs to the class of dissipative dynamics (Willems, 1972; Hill and Moylan, 1980).

\section{Problem statement}

Consider the following class of systems (Efimov and Aleksandrov, 2019):

$$
\dot{x}(t)=A_{0} x(t)+\sum_{j=1}^{M} A_{j} f^{j}(x(t))+d(t), t \geq 0,
$$

$y(t)=h(x(t))=\left[x^{\top}(t) C_{0}^{\top} f^{1}(x(t))^{\top} C_{1}^{\top} \ldots f^{M}(x(t))^{\top} C_{M}^{\top}\right]^{\top}$, where $x(t)=\left[x_{1}(t) \ldots x_{n}(t)\right]^{\top} \in \mathbb{R}^{n}$ is the state vector, $x(0) \in \mathbb{R}^{n} ; y(t) \in \mathbb{R}^{p}$ is the output signal, $p=\sum_{k=0}^{M} p_{k}$ and
$C_{k} \in \mathbb{R}^{p_{k} \times n}$ for $k=\overline{0, M}$; and $d(t) \in \mathbb{R}^{n}$ is the external perturbation, $d \in \mathcal{L}_{\infty}^{n} ; f^{j}(x)=\left[f_{1}^{j}\left(x_{1}\right) \ldots f_{n}^{j}\left(x_{n}\right)\right]^{\top}, j=$ $\overline{1, M}$ are the continuous functions ensuring existence and uniqueness of solutions of the system (3) in the forward time at least locally, the matrices $A_{k} \in \mathbb{R}^{n \times n}$ for $k=\overline{0, M}$.

In this paper, it is assumed that if the upper limit of a summation or a sequence is smaller than the lower one, then the corresponding terms (conditions) have to be omitted.

Assumption 1 For any $i=\overline{1, n}, j=\overline{1, M}$ :

$$
s f_{i}^{j}(s)>0 \quad \forall s \in \mathbb{R} \backslash\{0\} .
$$

In the assumption above it is stated that all nonlinearities belong to a sector and may take zero values at zero only, which is a usual characteristic of nonlinearities in Persidskii systems (Persidskii, 1969; Kazkurewicz and Bhaya, 1999) (if $A_{r}=0$ for all $r=\overline{0, M-1}$, then we recover the system studied by Persidskii in the conventional framework (Persidskii, 1969)). Then, after a proper re-indexing and decomposition of $f^{j}$, there exists $m \in\{0, \ldots, M\}$ such that for all $i=\overline{1, n}, z=\overline{1, m}$ :

$$
\lim _{s \rightarrow \pm \infty} f_{i}^{z}(s)= \pm \infty
$$

and there exists $\mu \in\{m, \ldots, M\}$ such that for all $i=\overline{1, n}$, $z=\overline{1, \mu}$ :

$$
\lim _{s \rightarrow \pm \infty} \int_{0}^{s} f_{i}^{z}(\sigma) d \sigma=+\infty
$$

Thus, some of the nonlinearities are radially unbounded, and $m=0$ corresponds to the case when all nonlinearities are bounded (at least for negative or positive argument); some of these nonlinearities have unbounded integrals, and clearly if $m>0$, then all radially unbounded nonlinearities also have unbounded integrals, thus $\mu \geq m$ due to the introduced sector condition.

For an output representing a part of the state vector (or after a change of coordinates), (3) is in the Lur'e form (Arcak and Teel, 2002; Sarkans and Logemann, 2015), then Assumption 1 is a variant of the conventional sector condition used in the absolute stability theory (Liberzon, 2006; Yakubovich, 2002):

$$
\underline{k}_{j} s^{2} \leq s f_{i}^{j}(s) \leq \bar{k}_{j} s^{2} \quad \forall s \in \mathbb{R}
$$

for any $i=\overline{1, n}$ and $j=\overline{1, M}$, where $0 \leq \underline{k}_{j}<\bar{k}_{j} \leq+\infty$ parameterize the nonlinearity. Note that if the latter restrictions are initially stated, by introducing new nonlinearities:

$$
\tilde{f}^{j}(x)=\hat{k}_{j}\left(f^{j}(x)-\tilde{k}_{j} x\right), j=\overline{1, M}
$$

for any $\hat{k}_{j}>0$ and $\tilde{k}_{j}<\underline{k}_{j}$, Assumption 1 can be recovered for $\tilde{f}^{j}(x)$, and the system saves the form (3) for properly recalculated matrices.

Our goal is to propose a constructive approach to check ISS, iISS, OSS and IOSS properties of (3). 


\section{$4 \quad$ Stability conditions}

The main result of this paper is as follows:

Theorem 3 Let Assumption 1 be satisfied and there exist $P=P^{\top} \in \mathbb{R}^{n \times n} ; \Xi^{k} \in \mathbb{D}_{+}^{n}$ for $k=\overline{0, M} ; \Lambda^{j} \in \mathbb{D}_{+}^{n}$ for $j=\overline{1, M} ; \Upsilon_{s, j} \in \mathbb{D}_{+}^{n}$ for $s=\overline{0, M-1}$ and $j=\overline{s+1, M}$, $\Gamma=\Gamma^{\top}>0, \varsigma \in \mathbb{R}$ and $\chi \geq 0$ such that

$$
\begin{gathered}
P \geq 0, P+\varsigma \sum_{z=1}^{\mu} \Lambda^{z}>0 ; Q \leq 0 ; \\
\sum_{k=0}^{M} \Xi^{k}+2 \sum_{s=0}^{M-1} \sum_{j=s+1}^{M} \Upsilon_{s, j}>0
\end{gathered}
$$

where

$$
\begin{gathered}
Q=\left[\begin{array}{cccccc}
Q_{1,1} & Q_{1,2} & Q_{1,3} & \cdots & Q_{1, M+1} & P \\
Q_{1,2}^{\top} & Q_{2,2} & Q_{2,3} & \cdots & Q_{2, M+1} & \Lambda^{1} \\
Q_{1,3}^{\top} & Q_{2,3}^{\top} & Q_{3,3} & \cdots & Q_{3, M+1} & \Lambda^{2} \\
\vdots & \vdots & \vdots & \ddots & \vdots & \vdots \\
Q_{1, M+1}^{\top} & Q_{2, M+1}^{\top} & Q_{3, M+1}^{\top} & \cdots & Q_{M+1, M+1} & \Lambda^{M} \\
P & \Lambda^{1} & \Lambda^{2} & \cdots & \Lambda^{M} & -\Gamma
\end{array}\right] \\
Q_{1,1}=A_{0}^{\top} P+P A_{0}+\Xi^{0}-\chi C_{0}^{\top} C_{0} ; \\
Q_{j+1, j+1}=A_{j}^{\top} \Lambda^{j}+\Lambda^{j} A_{j}+\Xi^{j}-\chi C_{j}^{\top} C_{j}, j=\overline{1, M} ; \\
Q_{1, j+1}=P A_{j}+A_{0}^{\top} \Lambda^{j}+\Upsilon_{0, j}, j=\overline{1, M} ; \\
Q_{s+1, j+1}=A_{s}^{\top} \Lambda^{j}+\Lambda^{s} A_{j}+\Upsilon_{s, j}, s=\overline{1, M-1}, j=\overline{s+1, M} .
\end{gathered}
$$

Then the system (3) is

$-i I S S$ for $\chi=0$

- ISS provided that $\chi=0$ and the last condition in (4) is strengthened to

$$
\begin{cases}\Xi^{0}>0 & m=0 \\ \sum_{k=0}^{m} \Xi^{k}+2 \sum_{s=0}^{m} \sum_{j=s+1}^{m} \Upsilon_{s, j}>0 & m>0\end{cases}
$$

- IOSS if the conditions of ISS are verified with some $\chi>0$.

PROOF. Consider a candidate Lyapunov function

$$
V(x)=x^{\top} P x+2 \sum_{j=1}^{M} \sum_{i=1}^{n} \Lambda_{i}^{j} \int_{0}^{x_{i}} f_{i}^{j}(s) d s,
$$

where $\Lambda_{i}^{j} \in \mathbb{R}_{+}$is $i^{\text {th }}$ element on the main diagonal of $\Lambda^{j}$. Note that $V(x)$ is positive definite and radially unbounded due to the properties of the nonlinear functions, introduced in Assumption 1, and the properties of the matrix $P$ specified in (4): by Finsler's lemma, existence of $\varsigma \in \mathbb{R}$ such that $P+\varsigma \sum_{z=1}^{\mu} \Lambda^{z}>0$ implies that $x^{\top} P x>0$ on the subspace where $x^{\top}\left(\sum_{z=1}^{\mu} \Lambda^{z}\right) x=0$ with $x \neq 0$, where $\mu \geq m$ indicates the nonlinearities with unbounded integrals. Then

$$
\begin{aligned}
\dot{V}= & \dot{x}^{\top} P x+x^{\top} P \dot{x}+2 \sum_{j=1}^{M} \sum_{i=1}^{n} \Lambda_{i}^{j} f_{i}^{j}\left(x_{i}\right) \dot{x}_{i} \\
= & \dot{x}^{\top} P x+x^{\top} P \dot{x}+2 \sum_{j=1}^{M} \dot{x}^{\top} \Lambda^{j} f^{j}(x) \\
= & x^{\top}\left(A_{0}^{\top} P+P A_{0}\right) x+\left(\sum_{j=1}^{M} f^{j}(x)^{\top} A_{j}^{\top}\right) P x \\
& +x^{\top} P \sum_{j=1}^{M} A_{j} f^{j}(x)+2 x^{\top} P d \\
& +2 \sum_{j=1}^{M}\left\{x^{\top} A_{0}^{\top} \Lambda^{j} f^{j}(x)+d^{\top} \Lambda^{j} f^{j}(x)\right. \\
& \left.+\left(\sum_{s=1}^{M} f^{s}(x)^{\top} A_{s}^{\top}\right) \Lambda^{j} f^{j}(x)\right\} \\
= & \xi^{\top} \tilde{Q} \xi+2 d^{\top} P x+2 d^{\top} \sum_{j=1}^{M} \Lambda^{j} f^{j}(x),
\end{aligned}
$$

where $\xi=\left[x^{\top} f^{1}(x)^{\top} \ldots f^{M}(x)^{\top}\right]^{\top}$ and

$$
\tilde{Q}=\left[\begin{array}{cccc}
A_{0}^{\top} P+P A_{0} & P A_{1}+A_{0}^{\top} \Lambda^{1} & \cdots & P A_{M}+A_{0}^{\top} \Lambda^{M} \\
A_{1}^{\top} P+\Lambda^{1} A_{0} & A_{1}^{\top} \Lambda^{1}+\Lambda^{1} A_{1} & \cdots & A_{1}^{\top} \Lambda^{M}+\Lambda^{1} A_{M} \\
\vdots & \vdots & \ddots & \vdots \\
A_{M}^{\top} P+\Lambda^{M} A_{0} & \Lambda^{M} A_{1}+A_{M}^{\top} \Lambda^{1} & \cdots & A_{M}^{\top} \Lambda^{M}+\Lambda^{M} A_{M}
\end{array}\right] .
$$

Therefore, under (4) we obtain

$$
\begin{aligned}
\dot{V}= & {\left[\begin{array}{l}
\xi \\
d
\end{array}\right]^{\top} Q\left[\begin{array}{l}
\xi \\
d
\end{array}\right]-x^{\top} \Xi^{0} x } \\
& -\sum_{j=1}^{M} f^{j}(x)^{\top} \Xi^{j} f^{j}(x)-2 \sum_{j=1}^{M} x^{\top} \Upsilon_{0, j} f^{j}(x) \\
& -2 \sum_{s=1}^{M-1} \sum_{j=s+1}^{M} f^{s}(x)^{\top} \Upsilon_{s, j} f^{j}(x) \\
& +\chi y^{\top} y+d^{\top} \Gamma d \\
\leq & -x^{\top} \Xi^{0} x-\sum_{j=1}^{M} f^{j}(x)^{\top} \Xi^{j} f^{j}(x) \\
& -2 \sum_{j=1}^{M} x^{\top} \Upsilon_{0, j} f^{j}(x) \\
& -2 \sum_{s=1}^{M-1} \sum_{j=s+1}^{M} f^{s}(x)^{\top} \Upsilon_{s, j} f^{j}(x)+\chi y^{\top} y+d^{\top} \Gamma d,
\end{aligned}
$$

which implies the desired iISS property for $\chi=0$ by The- 
orem 1. To ensure the ISS property, according to Theorem 1 , the function of $x$ in the right-hand side of the last inequality has to be radially unbounded, which is guaranteed by the introduced conditions:

$$
\chi=0, \sum_{k=0}^{m} \Xi^{k}+2 \sum_{s=0}^{m} \sum_{j=s+1}^{m} \Upsilon_{s, j}>0
$$

since only the first $m$ nonlinearities and the quadratic term are radially unbounded. Finally, by Theorem 2 the system is IOSS if these restrictions are verified for $\chi>0$.

In this result, the matrices $P$ and $\Lambda^{j}$ for $j=\overline{1, M}$ serve as solutions of the Lyapunov equation for the matrices $A_{0}$ and $A_{j}$ for $j=\overline{1, M}$, respectively. Note that if $P \in \mathbb{D}_{+}^{n}$, then the condition on positive definiteness of $V$ can be formulated as

$$
P+\sum_{z=1}^{\mu} \Lambda^{z}>0
$$

The matrices $\Xi^{k}$ for $k=\overline{0, M}$ are used in $Q$ in order to relax the stability requirements for each matrix $A_{k}$ for $k=$ $\overline{0, M}$ by looking for their total influence on the system performance (i.e., the imposed condition is $\sum_{k=0}^{M} \Xi^{k}>$ 0 , hence, the matrices $A_{k}, k=\overline{0, M}$ are not necessary Hurwitz).

The Lyapunov function $V(x)$ used in the proof is similar to one applied for Lur'e systems (Yakubovich et al., 2004; Hill and Bergen, 1982), but for $M>1$ a decomposition of nonlinearities as in (3) allows us to make a finer analysis for positive definiteness of $V$ and negative definiteness of $\dot{V}$. For example, the matrices $\Upsilon_{s, j}$ for $s=\overline{0, M-1}$ and $j=\overline{s+1, M}$ are introduced in order to reduce the conservatism of the condition $Q \leq 0$ for the case $M>1$, since all cross-terms of the form $x_{i} f_{i}^{j}\left(x_{i}\right)$ or $f_{i}^{j}\left(x_{i}\right) f_{i}^{s}\left(x_{i}\right)$ with $i=\overline{1, n}$, which appear in the off-diagonal blocks of $Q$, should not be considered as "perturbations" in verification of the restriction $Q \leq 0$ provided that they have a non-positive multiplier due to the sector properties of the functions $f_{i}^{j}$. The inclusion of $\Upsilon_{s, j}$ into consideration is an important feature of (4).

Remark 1 The conditions of ISS for the case $m=0$ can be relaxed to

$$
\Xi^{0}+2 \sum_{j=1}^{\varrho} \Upsilon_{0, j}>0
$$

under an additional hypothesis that the functions $x_{i} f_{i}^{j}\left(x_{i}\right)$ are radially unbounded for all $i=\overline{1, n}$ and $j=\overline{1, \varrho}$ with some $\varrho \in\{1, \ldots, M\}$ (similarly, the case with the unbounded cross-terms $f^{s}(x)^{\top} \Upsilon_{s, j} f^{j}(x)$ for $s=\overline{1, m}$ and $j=\overline{1, \varrho}$ with $\varrho>m>0$ can be treated).

Remark 2 We can also relax the conditions on positiveness of the matrices $\Lambda^{z}$ for $z=\overline{1, \mu}$ if we assume, for example, that there exist $\kappa_{z}>0$ such that

$$
\left\|f^{z}(x)\right\|^{2} \geq \kappa_{z}\|x\|^{2}
$$

for all $x \in \mathbb{R}^{n}$ and all $z=\overline{1, \mu}$. Then $\Lambda^{j} \in \mathbb{D}^{n}$ for $=\overline{1, \mu}$ and $\Lambda^{j} \in \mathbb{D}_{+}^{n}$ for $=\overline{\mu+1, M}$, while the corresponding LMIs in (4) can be replaced with the following ones:

$$
\begin{gathered}
P+\sum_{z=1}^{\mu} \kappa_{z} \min _{i=\overline{1, n}} \Lambda_{i}^{z} I_{n}>0 ; Q \leq 0 ; \\
\Theta \geq 0, \Theta+\sum_{k=\mu+1}^{M} \Xi^{k}+2 \sum_{s=0}^{M-1} \sum_{j=s+1}^{M} \Upsilon_{s, j}>0,
\end{gathered}
$$

where $\Theta=\Xi^{0}+\sum_{z=1}^{\mu} \kappa_{z} \min _{i=\overline{1, n}} \Xi_{i}^{z} I_{n}$. Of course, similar relaxations can also be imposed if one nonlinearity has a higher amplitude than another, e.g.,

$$
\left\|f^{z_{1}}(x)\right\|^{2} \geq \kappa_{z_{1}, z_{2}}\left\|f^{z_{2}}(x)\right\|^{2}
$$

for some $z_{1} \neq z_{2} \in\{1, \ldots, M\}$ and some $\kappa_{z_{1}, z_{2}}>0$ (the same for the cross terms $x^{\top} \Upsilon_{0, j} f^{j}(x)$ with $\left.j=\overline{1, M}\right)$.

Remark 3 In the ISS case, for $j=\overline{\mu+1, M}$ the sector requirement on $f_{i}^{j}$ introduced in Assumption 1 can be relaxed as follows:

$$
s f_{i}^{j}(s) \geq 0 \quad \forall s \in \mathbb{R}
$$

with $i=\overline{1, n}$. We can also extend such a consideration for all $j=\overline{1, M}$, but then we have to carefully analyze the issues with the absence of additional equilibria, or consider the multistability case (bi-stability, presence of limit cycles or other oscillating modes). Practical ISS conditions can be studied in the framework if, for example, we suppose in Assumption 1 that for all $j=\overline{1, M}$

$$
s f_{i}^{j}(s)>0 \quad \forall s \in \mathbb{R} \backslash\left[\underline{s}_{i}, \bar{s}_{i}\right]
$$

for any $i=\overline{1, n}$ with some $-\infty<\underline{s}_{i}<0<\bar{s}_{i}<+\infty$ (then it should be $P>0$ and $\Xi^{0}>0$ ).

Remark 4 It is straightforward to conclude that in order to satisfy the condition $Q \leq 0$ the matrices $A_{j}^{\top} \Lambda^{j}+\Lambda^{j} A_{j}$, which appear on the main diagonal of $Q$ for $j=\overline{1, M}$, have to be nonnegative definite also. Recall that $\Lambda^{j}$ are diagonal matrices, then for a stable Metzler matrix $A_{j}$ (a matrix with all nonnegative elements outside of the main diagonal) existence of such a diagonal matrix $\Lambda^{j}$ is necessary and sufficient for the stability. Note also that if in the system (3) the matrices $A_{k}$ for $k=\overline{0, M}$ are Metzler, then the corresponding terms $\sum_{i=1}^{n} \Lambda_{i}^{j} \int_{0}^{x_{i}} f_{i}^{j}(s) d s$ in the Lyapunov function can be replaced by $\sum_{i=1}^{n} \Lambda_{i}^{j}\left|x_{i}\right|$ (Persidskii, 1969), and the Lyapunov function can be simplified to the form (in such a case the integrals $\int_{0}^{x_{i}} f_{i}^{j}(s) d s$ can be bounded):

$$
V(x)=\sum_{i=1}^{n} \Lambda_{i}\left|x_{i}\right|
$$

with $P=0$ and $\Lambda \in \mathbb{D}_{+}^{n}$, whose derivative can be rewritten as follows $\left(\right.$ denote $\left.\operatorname{sign}(x)=\left[\operatorname{sign}\left(x_{1}\right) \ldots \operatorname{sign}\left(x_{n}\right)\right]^{\top}\right)$ :

$\dot{V}=\dot{x}^{\top} \Lambda \operatorname{sign}(x)=\left(A_{0} x+\sum_{j=1}^{M} A_{j} f^{j}(x)+d\right)^{\top} \Lambda \operatorname{sign}(x)$. 
Thus, if the following linear programming problem has a solution with respect to $\Lambda=\operatorname{diag}\{\lambda\}$ and $\xi^{k}=\left[\xi_{1}^{k} \ldots \xi_{n}^{k}\right]^{\top} \in$ $\mathbb{R}^{n}, k=\overline{0, M}$ :

$$
A_{k}^{\top} \lambda=-\xi^{k} \leq 0, k=\overline{0, M} ; \quad \sum_{k=0}^{M} \xi^{k}>0,
$$

then

$$
\dot{V} \leq-\sum_{i=1}^{n} \xi_{i}^{0}\left|x_{i}\right|-\sum_{j=1}^{M} \sum_{i=1}^{n} \xi_{i}^{j}\left|f_{i}^{j}\left(x_{i}\right)\right|+\sum_{i=1}^{n} \Lambda_{i}\left|d_{i}\right|,
$$

and the same stability conclusions can be deduced. The shortage of this approach is that in such a case all matrices $A_{k}$ for $k=\overline{0, M}$ have to possess a common Lyapunov function.

The statement of Theorem 3 and the remarks above describe the basic ideas of the approach and various relaxations or auxiliary results that can be obtained around (these developments are left for future research). Let us also formulate the conditions to check in the OSS case, which can also be useful for stability analysis:

Corollary 1 Let all conditions of Theorem 3 be satisfied for $\Gamma=0$ and

$$
Q=\left[\begin{array}{ccc}
Q_{1,1} & \cdots & Q_{1, M+1} \\
\vdots & \ddots & \vdots \\
Q_{1, M+1}^{\top} & \cdots & Q_{M+1, M+1}
\end{array}\right]
$$

where the elements of the matrix $Q$ are given in Theorem 3. Then the system (3) with $d(t)=0$ for all $t \geq 0$ is

- globally asymptotically stable with $\chi=0$;

- OSS provided that $\chi>0$ and the last condition in (4) is strengthened to

$$
\sum_{k=0}^{m} \Xi^{k}+2 \sum_{s=0}^{m} \sum_{j=s+1}^{m} \Upsilon_{s, j}>0
$$

PROOF. The proof follows exactly the same arguments as one of Theorem 3, then the result is a consequence of Theorem 2.

\section{Implementation with the use of Euler method}

For the conventional Persidskii system, the issues of application for discretization of solutions of the explicit Euler method have been analyzed in (Aleksandrov and Zhabko, 2010; Aleksandrov et al., 2012), and these results have been developed to the Persidskii systems with delays in (Aleksandrov and Aleksandrova, 2018). Application of the explicit and the implicit Euler methods to the extended class of Persidskii systems (3) with $d(t)=0$ for all $t \geq 0$ is considered in this section.

Denote the right-hand side of the system (3) by

$$
F(x)=A_{0} x+\sum_{j=1}^{M} A_{j} f^{j}(x),
$$

and assume that the system (3) admits the zero solution to be asymptotically stable. We will look for conditions of the preservation of the asymptotic stability after discretization of (3) using the Lyapunov function $V(x)$ constructed in the proof of Theorem 3 .

First, consider an application of the explicit Euler method:

$$
z(k+1)=z(k)+h F(z(k)), z(0)=x_{0} \in \mathbb{R}^{n},
$$

where $z(k) \in \mathbb{R}^{n}$ is an estimate of the solution $x\left(h k, x_{0}, 0\right)$ of the system $(3), h$ is a digitization step and $k=0,1, \ldots$ is the iteration number.

Theorem 4 Let the functions $f^{j}(x), j=\overline{1, M}$ be locally Lipschitz continuous. If the conditions of Corollary 1 are fulfilled with $\chi=0$ and $\Xi^{0}>0$, then for any $H>0$ there exists a constant $h_{0}>0$ such that the zero solution of (5) is asymptotically stable for all $h \in\left(0, h_{0}\right)$ and all initial conditions $x_{0} \in \Omega_{H}=\left\{x \in \mathbb{R}^{n}: V(x)<H\right\}$.

PROOF. Applying the approach proposed in (Aleksandrov and Zhabko, 2010) for conventional Persidskii systems and using the structure of the Lyapunov function proposed in Theorem 3 , it can be shown that for any $H>0$, one can choose numbers $a>0$ and $h_{0}>0$ such that

$$
V(z(k+1))-V(z(k)) \leq-a h\|z(k)\|^{2}
$$

for all $z(k) \in \Omega_{H}$ and $h \in\left(0, h_{0}\right)$, this implies the desired result.

Next, consider an application of the implicit Euler method:

$$
z(k+1)=z(k)+h F(z(k+1)), z(0)=x_{0} \in \mathbb{R}^{n} .
$$

Theorem 5 Let the functions $f^{j}(x)$ be continuously differentiable and $\frac{\partial f^{j}(x)}{\partial x} \geq 0$ for all $x \in \mathbb{R}^{n}, j=\overline{1, M}$. If the conditions of Corollary 1 are fulfilled with $\chi=0$, then for any $H>0$ there exists a constant $h_{0}>0$ such that for all $h \in\left(0, h_{0}\right)$ and all initial conditions $x_{0} \in \Omega_{H}$ :

(i) the solution $z(k)$ of (7) is defined for all $k=0,1, \ldots$;

(ii) the zero solution of (7) is asymptotically stable.

PROOF. Let $V(x)$ be the Lyapunov function constructed in the proof of Theorem 3. Using the Taylor expansion theorem with Lagrange remainder we obtain

$V(z(k+1))-V(z(k))=h\left(\frac{\partial V(z(k+1))}{\partial x}\right)^{\top} F(z(k+1))$ 


$$
-\frac{1}{2} \Delta z(k)^{\top} \frac{\partial^{2} V\left(z(k+1)-\theta_{k} \Delta z(k)\right)}{\partial x^{2}} \Delta z(k),
$$

where $\Delta z(k)=z(k+1)-z(k)$ and $\theta_{k} \in(0,1)$. It should be noted that

for any $x \in \mathbb{R}^{n}$. Hence,

$$
\frac{\partial^{2} V(x)}{\partial x^{2}} \geq 0
$$

$$
\begin{gathered}
V(z(k+1))-V(z(k)) \leq h\left(\frac{\partial V(z(k+1))}{\partial x}\right)^{\top} F(z(k+1)) \\
\leq-h z^{\top}(k+1) \Xi^{0} z(k+1) \\
-h \sum_{j=1}^{M} f^{j}(z(k+1))^{\top} \Xi^{j} f^{j}(z(k+1)),
\end{gathered}
$$

and unlike (6), the estimate (8) is valid for all $h>0$ and all $z(k+1) \in \mathbb{R}^{n}$. Therefore, if there is a solution $z(k+1)$ to $(7)$ for any $z(k)$, then for any step $h$ the discrete-time system (7) is globally converging to the origin.

Consider the conditions of existence of $z(k+1)$ on each iteration. Let a number $H>0$ be given. From (8) it follows that if a solution $z(k)$ of $(7)$ is defined for $k=\overline{0, l}$ with some $l>0$ and $x_{0} \in \Omega_{H}$, then $z(k) \in \Omega_{H}$ for $k=\overline{0, l}$. It is worth mentioning that $H$ is independent of $h$. Rewrite the system (7) as follows

$$
\eta=h F(z(k)+\eta)
$$

where $\eta=z(k+1)-z(k)$. Consider (9) for $z(k) \in \Omega_{H}$ and $\|\eta\| \leq 2 \delta$, where $\delta=\sup _{x \in \Omega_{H}}\|x\|$. Choose $h_{0}>0$ such that

$$
\|h F(z(k)+\eta)\| \leq 2 \delta
$$

for all $0<h \leq h_{0}$, all $z(k) \in \Omega_{H}$ and $\|\eta\| \leq 2 \delta$. Hence, if $0<h \leq h_{0}$, then the function $h F(z(k)+\cdot): B(2 \delta) \rightarrow$ $B(2 \delta)=\left\{x \in \mathbb{R}^{n}:\|x\| \leq 2 \delta\right\}$ is continuous on the convex compact set $B(2 \delta)$. Using the Brouwer fixed-point theorem (Leborgne, 1982), we conclude that the system (9) admits a solution $\eta \in B(2 \delta)$ for any $z(k) \in \Omega_{H}$. In addition, from (8) it follows that (9) does not possess solutions with $\|\eta\|>2 \delta$.

Therefore, for any $x_{0} \in \Omega_{H}$ the corresponding solution $z(k)$ of $(7)$ is defined for all $k=0,1, \ldots$ and $z(k) \in \Omega_{H}$. Moreover, the estimate (8) implies that $\|z(k)\| \rightarrow 0$ as $k \rightarrow \infty$. This completes the proof.

The proof of theorems 4 and 5 shows that the approximation dynamics (5) and (7) keep the asymptotic stability property of the original system (3) under the same restrictions on the right-hand side as in Theorem 3 with the same Lyapunov function.

Comparing the results of theorems 4 and 5 it is worth to stress that the limit $h_{0}$ on the admissible discretization step $h$ for the implicit method comes form the conditions of existence of $z(k+1)$ in $(7)$ (this scheme is always stable under the hypotheses of Theorem 5), while for the explicit method (5) the discretization may loose its stability if the step $h$ is not sufficiently small. Theorem 5 also imposes additional conditions on monotonicity of the nonlinearities (i.e., $\frac{\partial f^{j}(x)}{\partial x} \geq 0$ for all $x \in \mathbb{R}^{n}$ ), but under less restrictive stability conditions (the requirement $\Xi^{0}>0$ is not introduced).

\section{Applications}

Consider the problem of robust stabilization for a nominal linear dynamical plant:

$$
\dot{x}=A x+B u+E d,
$$

where $x \in \mathbb{R}^{n}$ is the state vector; $d \in \mathbb{R}^{q}$ is the vector either representing the external perturbations, then $d \in \mathcal{L}_{\infty}^{q}$, or hiding the unmodeled nonlinearities, then we assume that

$$
\|d\|^{2} \leq \sum_{i=1}^{n} R_{i}^{0}\left|x_{i}\right|^{2}+R_{i}^{1}\left|x_{i}\right|^{1+\alpha}+R_{i}^{2}\left|x_{i}\right|^{1+\beta}
$$

where $\alpha \in(0,1)$ and $\beta>1$ are growth parameters, $R^{j} \in$ $\mathbb{D}_{+}^{n}$ for $j=\overline{0,2}$; and $u \in \mathbb{R}^{w}$ is the control input, $w \leq n ; A \in$ $\mathbb{R}^{n \times n}, B \in \mathbb{R}^{n \times w}$ and $E \in \mathbb{R}^{n \times q}$ are constant matrices.

We would like to synthesize a feedback $u=u(x)$ providing to (10) the ISS property for $d \in \mathcal{L}_{\infty}^{q}$ and the asymptotic stability property for the closed loop system under the disturbances satisfying (11).

Note that from the bound (11), the norm of the perturbation $\|d\|$ has a growth proportional to $\left|x_{i}\right|^{\frac{1+\alpha}{2}}$ close to the origin with $\frac{1+\alpha}{2}<1$ and to $\left|x_{i}\right|^{\frac{1+\beta}{2}}$ far outside with $\frac{1+\beta}{2}>1$. Therefore, (11) can represent any polynomial bounded nonlinearities, and (10) under (11) is a highly nonlinear system, then a linear feedback cannot solve the problem of its stabilization. Such a polynomial upper bound on disturbances is frequently used by fixed-time stabilization approaches (Polyakov et al., 2016).

Following (Aparicio et al., 2016, 2019) consider the control in the form:

$$
u(x)=K_{0}^{\top} x+K_{1}^{\top} f^{1}(x)+K_{2}^{\top} f^{2}(x),
$$

where $K_{0}, K_{1}, K_{2} \in \mathbb{R}^{n \times w}$ are the control gains to be designed, and $f^{j}(x)=\left[f_{1}^{j}\left(x_{1}\right) \ldots f_{n}^{j}\left(x_{n}\right)\right]^{\top}$ for $j=1,2$ are as before, where

$$
f_{i}^{1}\left(x_{i}\right)=\left|x_{i}\right|^{\alpha} \operatorname{sign}\left(x_{i}\right), f_{i}^{2}\left(x_{i}\right)=\left|x_{i}\right|^{\beta} \operatorname{sign}\left(x_{i}\right) .
$$

Note that (12) is a continuous function of $x$ since $\alpha>0$. Substituting this control in the system equations we obtain:

$$
\dot{x}=A_{0} x+A_{1} f^{1}(x)+A_{2} f^{2}(x)+E d,
$$

where $A_{0}=A+B K_{0}^{\top}, A_{1}=B K_{1}^{\top}$ and $A_{2}=B K_{2}^{\top}$. Clearly, this dynamics is in the Persidskii form similar to (3), then the time derivative of the Lyapunov function $V(x)$ can be presented as follows:

$$
\dot{V}=\xi^{\top} Q^{\prime} \xi+\gamma d^{\top} d,
$$


where $\xi=\left[x^{\top} f^{1}(x)^{\top} f^{2}(x)^{\top} d^{\top}\right]^{\top}$ and

$$
Q^{\prime}=\left[\begin{array}{cc} 
& P E \\
\tilde{Q} & \Lambda^{1} E \\
& \Lambda^{2} E \\
E^{\top} P E^{\top} \Lambda^{1} E^{\top} \Lambda^{2} & -\gamma I_{q}
\end{array}\right]
$$

with the matrix $\tilde{Q}$ given in the proof of Theorem 3 for $M=2$, and $\gamma>0$ is a tuning parameter. It is straightforward to check that the diagonal blocks $\tilde{Q}_{2,2}$ and $\tilde{Q}_{3,3}$ have maximally the rank equal to $w$, then they cannot ensure non-positive definiteness of $Q^{\prime}$ if $w<n$. Therefore, a direct application of Theorem 3 is not efficient, then let us adapt the stability arguments given in Theorem 3 to this scenario. To this end, note that the blocks $\tilde{Q}_{2,3}=A_{1}^{\top} \Lambda^{2}+\Lambda^{1} A_{2}$ and $\tilde{Q}_{3,2}=\tilde{Q}_{2,3}^{\top}$ also have the rank equal to $w$, then the only blocks to treat specially are

$$
\tilde{Q}_{1,2}=P A_{1}+A_{0}^{\top} \Lambda^{1}, \tilde{Q}_{1,3}=P A_{2}+A_{0}^{\top} \Lambda^{2}
$$

and their symmetric counterparts $\tilde{Q}_{2,1}$ and $\tilde{Q}_{3,1}$, respectively. Putting the corresponding terms out of the matrix $Q^{\prime}$ we obtain:

$$
\begin{aligned}
\dot{V}= & \xi^{\top} \hat{Q} \xi-x^{\top} \Xi^{0} x-2 \sum_{j=1}^{2} x^{\top} \Upsilon_{0, j} f^{j}(x)+\gamma d^{\top} d \\
& +2 \sum_{j=1}^{2} x^{\top}\left(P A_{j}+A_{0}^{\top} \Lambda^{j}+\Upsilon_{0, j}\right) f^{j}(x)
\end{aligned}
$$

where

$$
\hat{Q}=\left[\begin{array}{cccc}
A_{0}^{\top} P+P A_{0}+\Xi^{0} & 0 & 0 & P E \\
0 & A_{1}^{\top} \Lambda^{1}+\Lambda^{1} A_{1} & A_{1}^{\top} \Lambda^{2}+\Lambda^{1} A_{2} & \Lambda^{1} E \\
0 & A_{2}^{\top} \Lambda^{1}+\Lambda^{2} A_{1} & A_{2}^{\top} \Lambda^{2}+\Lambda^{2} A_{2} & \Lambda^{2} E \\
E^{\top} P & E^{\top} \Lambda^{1} & E^{\top} \Lambda^{2} & -\gamma I_{q}
\end{array}\right]
$$

and $\Xi^{0}, \Upsilon_{0,1}, \Upsilon_{0,2} \in \mathbb{D}_{+}^{n}$ are matrices, which will be selected later. As before, the elements on the main diagonal of $P A_{j}+A_{0}^{\top} \Lambda^{j}+\Upsilon_{0, j}$ are useful if they are negative, while other cross terms can be treated using Young's inequality:

$$
\begin{aligned}
& x_{i}\left|x_{k}\right|^{\alpha} \operatorname{sign}\left(x_{k}\right) \leq \frac{\left|x_{i}\right|^{1+\alpha}}{1+\alpha}+\frac{\alpha\left|x_{k}\right|^{1+\alpha}}{1+\alpha}, \\
& x_{i}\left|x_{k}\right|^{\beta} \operatorname{sign}\left(x_{k}\right) \leq \frac{\left|x_{i}\right|^{1+\beta}}{1+\beta}+\frac{\beta\left|x_{k}\right|^{1+\beta}}{1+\beta}
\end{aligned}
$$

for any $i \neq k=\overline{1, n}$, then all of them can be converted to the terms appeared on the main diagonal. Hence, if $\hat{Q}$ is non-positive definite and $P A_{j}+A_{0}^{\top} \Lambda^{j}+\Upsilon_{0, j}$ is diagonally dominant, i.e. if

$$
\begin{gathered}
\hat{Q} \leq 0, \\
1_{n}^{\top}\left[(1+\alpha) \delta\left(P A_{1}+A_{0}^{\top} \Lambda^{1}\right)+\alpha \chi\left(P A_{1}+A_{0}^{\top} \Lambda^{1}\right)\right. \\
\left.+\chi^{\top}\left(P A_{1}+A_{0}^{\top} \Lambda^{1}\right)+\Upsilon_{0,1}\right] \leq 0, \\
1_{n}^{\top}\left[(1+\beta) \delta\left(P A_{2}+A_{0}^{\top} \Lambda^{2}\right)+\beta \chi\left(P A_{2}+A_{0}^{\top} \Lambda^{2}\right)\right. \\
\left.+\chi^{\top}\left(P A_{2}+A_{0}^{\top} \Lambda^{2}\right)+\Upsilon_{0,2}\right] \leq 0,
\end{gathered}
$$

where $\delta(\mathcal{A}) \in \mathbb{D}^{n}$ contains the elements from the main diagonal of a matrix $\mathcal{A} \in \mathbb{R}^{n \times n}$ and $\chi(\mathcal{A})$ corresponds to the matrix with zeros on the main diagonal and absolute values of other elements of $\mathcal{A}$, then

$$
x^{\top}\left(P A_{j}+A_{0}^{\top} \Lambda^{j}+\Upsilon_{0, j}\right) f^{j}(x) \leq 0, j=1,2
$$

and, hence,

$$
\dot{V} \leq-x^{\top} \Xi^{0} x-2 \sum_{j=1}^{2} x^{\top} \Upsilon_{0, j} f^{j}(x)+\gamma d^{\top} d .
$$

Therefore, the following result has been proven:

Theorem 6 Let for the system (10) with the control (12) for the given gains $K_{0}, K_{1}, K_{2} \in \mathbb{R}^{n \times w}$ and powers $\alpha \in$ $(0,1), \beta>1$ there exist $P=P^{\top} \in \mathbb{R}^{n \times n} ; \Xi^{0} \in \mathbb{D}_{+}^{n}$; $\Lambda^{j} \in \mathbb{D}_{+}^{n}$ for $j=\overline{1,2} ; \Upsilon_{0, j} \in \mathbb{D}_{+}^{n}$ for $j=\overline{1,2}, \varsigma \in \mathbb{R}$ and $\gamma>0$ such that LMIs

$$
P \geq 0, P+\varsigma \sum_{z=1}^{2} \Lambda^{z}>0 ; \Xi^{0}+\sum_{j=1}^{2} 2 \Upsilon_{0, j}>0
$$

and (13) are satisfied. Then the system (10), (12) is ISS with respect to the disturbance $d \in \mathcal{L}_{\infty}^{q}$, and if additionally the estimate (11) holds and

$$
\begin{aligned}
& \Xi^{0} \geq \gamma R_{0}, \Upsilon_{0, j} \geq \gamma R_{j}, j=\overline{1,2}, \\
& \Xi^{0}-\gamma R_{0}+\sum_{j=1}^{2} 2 \Upsilon_{0, j}-\gamma R_{j}>0,
\end{aligned}
$$

then (10), (12) is globally asymptotically stable.

PROOF. For stability analysis of the closed-loop system $(10),(12)$ the same Lyapunov function $V$ is used as in Theorem 3 , and the proof of positive definiteness of $V$ follows the same arguments. The negative definiteness of the time derivative of $V$ for (10), (12) is analyzed above, and the ISS property follows. For the disturbance $d$ satisfying (11) we obtain:

$\dot{V} \leq-x^{\top}\left(\Xi^{0}-\gamma R_{0}\right) x-\sum_{j=1}^{2} x^{\top}\left(2 \Upsilon_{0, j}-\gamma R_{j}\right) f^{j}(x)$,

which under the introduced LMIs implies the required asymptotic stability property.

Remark 5 In order to assure that the last two LMIs in (13) are satisfied, an admissible condition is to have the matrix $A_{0}$ to be diagonally dominant (indeed, $P A_{j}$ is of rank $w$ and the only possible full rank matrix is $\left.A_{0}^{\top} \Lambda^{j}, j=1,2\right)$. If it is not the case, e.g., A represents a chain of integrators and $B$ is a vector that maps the scalar control to the last state variable $x_{n}$ only, then a change of coordinates $z=S x$ can be used as in (Aparicio et al., 2016, 2019), with posterior design of the control in the new coordinates $z$. 
Note that if in the formulation of Theorem 6 the latter LMIs are valid in the strict sense:

$$
\Xi^{0}>\gamma R_{0}, \Upsilon_{0, j}>\gamma R_{j}, j=\overline{1,2},
$$

then an exponential convergence of (10), (12) can be guaranteed under (11). Moreover, it is a well-known fact that the presence in the control (12) of the powers $\alpha$ and $\beta$, which are smaller and higher than one, respectively, may lead even to a fixed-time convergence rate (Polyakov et al., 2015; Lopez-Ramirez et al., 2018, 2019). The main drawback of Theorem 6 is that the control gains $K_{0}, K_{1}$ and $K_{2}$ are supposed to be given (the powers $\alpha$ and $\beta$ come from (11)). This requirement can be relaxed under an additional mild condition:

Corollary 2 Let for the system (10) with the control (12) the gains be chosen as $K_{0}=P^{-1} M_{0}, K_{j}=\left(\Lambda^{j}\right)^{-1} M_{j}, j=$ 1,2 with $\alpha \in(0,1), \beta>1$, where $P \in \mathbb{D}_{+}^{n}$ and $M_{s} \in \mathbb{R}^{n \times w}$, $s=\overline{0,2}$ are the solutions of the LMIs:

$$
\begin{gathered}
P>0, \Lambda^{j}>0, j=\overline{1,2} ; \Xi^{0}+\sum_{j=1}^{2} \Upsilon_{0, j}>0, \\
1_{n}^{\top}\left[(1+\alpha) \delta\left(\Phi_{1}\right)+\alpha \chi\left(\Phi_{1}\right)+\chi^{\top}\left(\Phi_{1}\right)\right] \leq 0, \\
\left.\begin{array}{cccc}
1_{n}^{\top}\left[(1+\beta) \delta\left(\Phi_{2}\right)+\beta \chi\left(\Phi_{2}\right)+\chi^{\top}\left(\Phi_{2}\right)\right] \leq 0, \\
\Pi & 0 & 0 & E \\
0 & B M_{1}^{\top}+M_{1} B^{\top} & B M_{2}^{\top}+M_{1} B^{\top} & E \\
0 & B M_{1}^{\top}+M_{2} B^{\top} & B M_{2}^{\top}+M_{2} B^{\top} & E \\
E^{\top} & E^{\top} & E^{\top} & -\gamma I_{q}
\end{array}\right] \leq 0,
\end{gathered}
$$

for

$$
\begin{gathered}
\Pi=P^{-1} A^{\top}+A P^{-1}+M_{0} B^{\top}+B M_{0}^{\top}+\Xi^{0}, \\
\Phi_{1}=B M_{1}^{\top}+P^{-1} A^{\top}+M_{0} B^{\top}+\Upsilon_{0,1}, \\
\Phi_{2}=B M_{2}^{\top}+P^{-1} A^{\top}+M_{0} B^{\top}+\Upsilon_{0,2},
\end{gathered}
$$

$\Xi^{0} \in \mathbb{D}_{+}^{n} ; \Lambda^{j} \in \mathbb{D}_{+}^{n}$ for $j=\overline{1,2} ; \Upsilon_{0, j} \in \mathbb{D}_{+}^{n}$ for $j=\overline{1,2}$, and $\gamma>0$. Then the system (10), (12) is ISS with respect to the disturbance $d \in \mathcal{L}_{\infty}^{q}$, and if additionally the estimate (11) holds and

$$
\begin{gathered}
P \Xi^{0} P \geq \gamma R_{0}, P \Upsilon_{0, j} \Lambda^{j} \geq \gamma R_{j}, j=\overline{1,2}, \\
P \Xi^{0} P-\gamma R_{0}+\sum_{j=1}^{2} 2 \Upsilon_{0, j} \Lambda^{j}-\gamma R_{j}>0,
\end{gathered}
$$

then (10), (12) is globally asymptotically stable.

PROOF. Denote

$$
\Omega=\left[\begin{array}{cccc}
P & 0 & 0 & 0 \\
0 & \Lambda^{1} & 0 & 0 \\
0 & 0 & \Lambda^{2} & 0 \\
0 & 0 & 0 & I_{q}
\end{array}\right],
$$

which is an invertible matrix due to the conditions of the corollary, then

$$
\begin{gathered}
\dot{V}=\left[\begin{array}{c}
x \\
f^{1}(x) \\
f^{2}(x) \\
d
\end{array}\right]^{\top} \Omega \bar{Q} \Omega\left[\begin{array}{c}
x \\
f^{1}(x) \\
f^{2}(x) \\
d
\end{array}\right]+\gamma d^{\top} d \\
-x^{\top} P \Xi^{0} P x-2 \sum_{j=1}^{2} x^{\top} P \Upsilon_{0, j} \Lambda^{j} f^{j}(x),
\end{gathered}
$$

where

$$
\begin{gathered}
\bar{Q}=\Omega^{-1} Q^{\prime} \Omega^{-1}+\left[\begin{array}{cccc}
\Xi^{0} & \Upsilon_{0,1} & \Upsilon_{0,2} & 0 \\
\Upsilon_{0,1} & 0 & 0 & 0 \\
\Upsilon_{0,2} & 0 & 0 & 0 \\
0 & 0 & 0 & 0
\end{array}\right] \\
=\left[\begin{array}{cccc}
\Pi & \Phi_{1} & \Phi_{2} & E \\
\Phi_{1}^{\top} & B M_{1}^{\top}+M_{1} B^{\top} & B M_{2}^{\top}+M_{1} B^{\top} & E \\
\Phi_{2}^{\top} & B M_{1}^{\top}+M_{2} B^{\top} & B M_{2}^{\top}+M_{2} B^{\top} & E \\
E^{\top} & E^{\top} & E^{\top} & -\gamma I_{q}
\end{array}\right] .
\end{gathered}
$$

Since $P$ is assumed to be diagonal (as well as $\Lambda^{1}$ and $\Lambda^{2}$ ), the terms corresponding to $\bar{Q}_{1,2}=\Phi_{1}$ and $\bar{Q}_{1,3}=\Phi_{2}$ can be treated similarly as $\tilde{Q}_{1,2}$ and $\tilde{Q}_{1,3}$ in Theorem 6 , which is formulated in the LMIs of the corollary, then we obtain:

$$
\dot{V}=-x^{\top} P \Xi^{0} P x-2 \sum_{j=1}^{2} x^{\top} P \Upsilon_{0, j} \Lambda^{j} f^{j}(x)+\gamma d^{\top} d,
$$

from which the desired conclusion follows.

The only restriction introduced in the last corollary is the diagonal structure of the matrix $P$, which allows the LMIs of Theorem 6 to be reformulated having the gains $K_{0}, K_{1}$ and $K_{2}$ as decision variables. For application, the LMIs of Corollary 2 can be applied to calculate the control gains, and next the LMIs of Theorem 6 can be used for these $K_{0}, K_{1}, K_{2}$ and a generic structure of $P$, in order to optimize the value of $\gamma$ and the possible tolerated disturbance satisfying (11).

Example 1 Let $n=3$ and $w=1$,

$$
A=\left[\begin{array}{ccc}
-1 & 2 & 0.1 \\
0 & 2 & -0.1 \\
0 & 0 & -1
\end{array}\right], B=\left[\begin{array}{l}
1 \\
2 \\
1
\end{array}\right], E=\left[\begin{array}{c}
-0.5 \\
-1 \\
-0.5
\end{array}\right] \text {, }
$$

then solving the proposed in Corollary 2 LMIs we obtain:

$$
\begin{gathered}
K_{0}=[0.0055,-3.9125,0.0394]^{\top}, \\
K_{1}=K_{2}=-[0.0136,0.0273,0.0136]^{\top}, \\
\alpha=0.5, \beta=1.5, \gamma=24.2386, \\
R_{0}=10^{-4} \times \operatorname{diag}\{[5,239,4]\},
\end{gathered}
$$




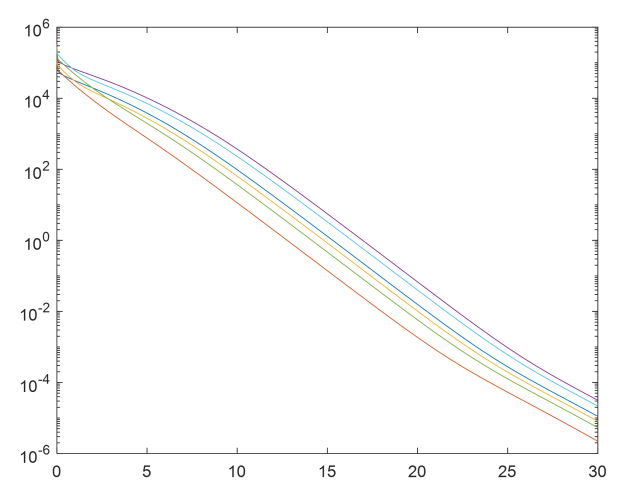

Fig. 1. The results of simulation

$$
\begin{aligned}
& R_{1}=10^{-4} \times \operatorname{diag}\{[1854,2618,1546]\}, \\
& R_{2}=10^{-4} \times \operatorname{diag}\{[1735,5964,1276]\} .
\end{aligned}
$$

The norm of the state on trajectories of the controlled system with six different initial conditions is shown in Fig. 1 in logarithmic scale.

Remark 6 Another application of the proposed approach for analysis of stability of an interval predictor is given in (Leurent et al., 2019). The result of Theorem 3 can also be used for investigation of stability of a generalized Lotka-Volterra equation with a support of mutualistic interactions (Efimov and Aleksandrov, 2019):

$$
\dot{x}(t)=\operatorname{diag}\{x(t)\}\left(b+A x(t)+A_{2} \varphi(x(t))\right), t \geq 0,
$$

where $x(t) \in \mathbb{R}_{+}^{n}$ is the vector of the populations of $n$ biological species, $x(0) \in \mathbb{R}_{+}^{n} ; b \in \mathbb{R}^{n}$ corresponds to the intrinsic birth or death rates of the species, $A \in \mathbb{R}^{n \times n}$ is the community matrix, and $A_{2} \in \mathbb{R}^{n \times n}$ is the mutualistic interaction strength between the species; $\varphi(x(t))=$ $\left[\varphi_{1}\left(x_{1}\right) \ldots \varphi_{n}\left(x_{n}\right)\right]^{\top}=\left[\frac{x_{1}}{r_{1}+x_{1}} \ldots \frac{x_{n}}{r_{n}+x_{n}}\right]^{\top}$ is the vector of Michaelis-Menten functions with $r=\left[r_{1} \ldots r_{n}\right]^{\top} \in \mathbb{R}^{n}$ being the half-saturation constants.

\section{Conclusions}

The framework of Persidskii systems is revisited and new conditions of ISS, iISS, IOSS and OSS properties are proposed, which are given in terms of LMIs. The proposed theory is applied to design a robustly stabilizing feedback. It is shown that Euler discretizations of such a class of Persidskii systems preserve stability of the solutions. Our further directions of research will include the analysis of feasibility of LMIs given in Section 4 and reformulation of the LMIs (4) and (13) to better take the features of the particular control and estimation problems into account. Another possible direction is analysis of conditions of stabilization by a bounded control.

\section{References}

Aleksandrov, A. and Aleksandrova, E. (2018). Delayindependent stability conditions for a class of nonlinear difference systems. Journal of the Franklin Institute, 355, 3367-3380.

Aleksandrov, A., Chen, Y., Platonov, A., and Zhang, L. (2012). Stability analysis and uniform ultimate boundedness control synthesis for a class of nonlinear switched difference systems. J. Differ. Equat. Appl., 18(9), 15451561.

Aleksandrov, A. and Zhabko, A. (2010). Preservation of stability under discretization of systems of ordinary differential equations. Siberian Math. J., 51(3), 383-395.

Altafini, C. (2013). Consensus problems on networks with antagonistic interactions. IEEE Transactions on Automatic Control, 58(4), 935-946.

Aparicio, A., Efimov, D., and Fridman, L. (2016). Stabilization of a triple integrator by a 3 -sign feedback. In Proc. 55th IEEE Conference on Decision and Control $(C D C)$. Las Vegas.

Aparicio, A., Fridman, L., and Efimov, D. (2019). Stabilization of systems with switchings on the axis of their coordinates and its input-to-state properties. Nonlinear Analysis: Hybrid Systems, 32, 10-18. doi: https://doi.org/10.1016/j.nahs.2018.10.008.

Arcak, M. and Teel, A. (2002). Input-to-state stability for a class of Lurie systems. Automatica J. IFAC, 38(11), 1945-1949.

Bacciotti, A. and Rosier, L. (2005). Lyapunov Functions and Stability in Control Theory. Springer, Berlin, 2nd edition.

Barbashin, E. (1961). On construction of Lyapunov functions for nonlinear systems. In Proc. 1st IFAC World Congress, 742-751. Moscow.

Dashkovskiy, S., Efimov, D., and Sontag, E. (2011). Input to state stability and allied system properties. Automation and Remote Control, 72(8), 1579-1614.

Efimov, D. and Aleksandrov, A. (2019). Robust stability analysis and implementation of Persidskii systems. In Proc. 58th IEEE Conference on Decision and Control $(C D C)$. Nice.

Efimov, D., Polyakov, A., and Aleksandrov, A. (2019). Discretization of homogeneous systems using Euler method with a state-dependent step. Automatica.

Efimov, D., Polyakov, A., Levant, A., and Perruquetti, W. (2017). Realization and discretization of asymptotically stable homogeneous systems. IEEE Transactions on Automatic Control, 62(11), 5962-5969.

Erickson, K. and Michel, A. (1985). Stability analysis of fixed-point digital filters using computer generated Lyapunov functions - Part I: Direct form and coupled form filters. IEEE Trans. on Circuits and Systems, 32, 113132.

Ferreira, L., Kaszkurewicz, E., and Bhaya, A. (2005). Solving systems of linear equations via gradient systems with discontinuous righthand sides: application to LS-SVM. IEEE Transactions on Neural Networks, 16(2), 501-505. doi:10.1109/TNN.2005.844091.

Hahn, W. (1967). Stability of Motion. Springer-Verlag, New York.

Halanay, A. (1966). Difierential Equations. Stability. Oscillations. Time Lags. Academic Press, London. 
Hill, D. and Bergen, A. (1982). Stability analysis of multimachine power networks with linear frequency dependent loads. IEEE Transactions on Circuits and Systems, 29(12), 840-848. doi:10.1109/TCS.1982.1085110.

Hill, D.J. and Moylan, P.J. (1980). Dissipative dynamical systems: basic input-output and state properties. $J$. Franklin Inst., 309(5), 327-357.

Hopfield, J. and Tank, D. (1986). Computing with neural circuits: a model. Science, 233, 625-633.

Hsu, L., Kaszkurewicz, E., and Bhaya, A. (2000). Matrixtheoretic conditions for the realizability of sliding manifolds. Systems \& Control Letters, 40(3), 145-152. doi: https://doi.org/10.1016/S0167-6911(00)00013-X.

Karny, M., Warwick, K., and Kurkova, V. (eds.) (1998). Dealing with Complexity: A Neural Networks Approach. Springer-Verlag, London.

Kazkurewicz, E. and Bhaya, A. (1999). Matrix Diagonal Stability in Systems and Computation. Birkhauser, Boston.

Khalil, H.K. (1996). Nonlinear Systems. NJ 07458. Prentice-Hall, Upper Saddle River.

LaSalle, J. and Lefchetz, S. (1961). Stability by Liapunov's Direct Method with Aplications. Academic Press, New York.

Leborgne, D. (1982). Calcul différentiel et géométrie. Puf, Paris.

Leurent, E., Efimov, D., Raïssi, T., and Perruquetti, W. (2019). Interval prediction for continuous-time systems with parametric uncertainties. In Proc. 58th IEEE Conference on Decision and Control (CDC). Nice.

Liberzon, M. (2006). Essays on the absolute stability theory. Autom Remote Control, 67, 1610-1644.

Lin, Y., Sontag, E.D., and Wang, Y. (1996). A smooth converse Lyapunov theorem for robust stability. SIAM Journal on Control and Optimization, 34(1), 124-160.

Lopez-Ramirez, F., Efimov, D., Polyakov, A., and Perruquetti, W. (2018). Fixed-time output stabilization and fixed-time estimation of the chain of integrators. International Journal of Robust and Nonlinear Control, 28(16), 4647-4665.

Lopez-Ramirez, F., Efimov, D., Polyakov, A., and Perruquetti, W. (2019). Conditions for fixed-time stability and stabilization of continuous autonomous systems. Systems \& Control Letters, 129(7), 26-35.

Lyapunov, A.M. (1992). Stability of motion: General problem. Internat. J. Control, 55(3), 520-790. Lyapunov Centenary issue.

Malkin, I. (1952). Theory of Stability of Motion, volume Translation Series: Physics and Mathematics. US Atomic Energy Commission.

Persidskii, S. (1969). Problem of absolute stability. Automation and Remote Control, 12, 1889-1895.

Polyakov, A., Efimov, D., and Perruquetti, W. (2015). Finite-time and fixed-time stabilization: Implicit lyapunov function approach. Automatica, 51, 332-340.

Polyakov, A., Efimov, D., and Perruquetti, W. (2016). Robust stabilization of MIMO systems in finite/fixed time. International Journal of Robust and Nonlinear Control, 26(1), 69-90.
Sarkans, E. and Logemann, H. (2015). Input-to-state stability of Lur'e systems. Math. Control Signals Syst, 27, 439-465.

Sontag, E. (1993). Recurrent networks. In Some Topics in Neural Networks and Control.

Sontag, E.D. (1998). Comments on integral variants of ISS. Systems Control Lett., 34(1-2), 93-100.

Sontag, E.D. (2001). The ISS philosophy as a unifying framework for stability-like behavior. In Nonlinear control in the year 2000, Vol. 2 (Paris), volume 259 of Lecture Notes in Control and Inform. Sci., 443-467. Springer, London.

van der Schaft, A. (1996). L $L_{2}$-gain and passivity techniques in nonlinear control, volume 218 of Lecture Notes in Control and Information Sciences. Springer-Verlag London Ltd., London.

Willems, J.C. (1972). Dissipative dynamical systems. I. General theory. Arch. Rational Mech. Anal., 45, 321351.

Yakubovich, V.A., Leonov, G.A., and Gelig, A.K. (2004). Stability of Stationary Sets in Control Systems with Discontinuous Nonlinearities. WORLD SCIENTIFIC. doi: $10.1142 / 5442$.

Yakubovich, V. (2002). Popov's method and its subsequent development. European Journal of Control, 8(3), 200208. doi:https://doi.org/10.3166/ejc.8.200-208.

Zhang, X. (2020). Robust integral sliding mode control for uncertain switched systems under arbitrary switching rules. Nonlinear Analysis: Hybrid Systems, 37, 100900. doi:https://doi.org/10.1016/j.nahs.2020.100900. 